\title{
Three-body recombination in a three-state Fermi gas with widely tunable interactions
}

\author{
J. H. Huckans, J. R. Williams, E. L. Hazlett, R. W. Stites, and K. M. O’Hara \\ Department of Physics, Pennsylvania State University, University Park, Pennsylvania 16802-6300, USA
}

(Dated: October 29, 2018)

\begin{abstract}
We investigate the stability of a three spin state mixture of ultracold fermionic ${ }^{6} \mathrm{Li}$ atoms over a range of magnetic fields encompassing three Feshbach resonances. For most field values, we attribute decay of the atomic population to three-body processes involving one atom from each spin state and find that the three-body loss coefficient varies by over four orders of magnitude. We observe high stability when at least two of the three scattering lengths are small, rapid loss near the Feshbach resonances, and two unexpected resonant loss features. At our highest fields, where all pairwise scattering lengths are approaching $a_{t}=-2140 a_{0}$, we measure a three-body loss coefficient $L_{3} \simeq 5 \times 10^{-22} \mathrm{~cm}^{6} / \mathrm{s}$ and a trend toward lower decay rates for higher fields indicating that future studies of color superfluidity and trion formation in a $\mathrm{SU}(3)$ symmetric Fermi gas may be feasible.
\end{abstract}

PACS numbers: 67.85.Lm, 34.50.-s, 67.85.-d, 03.75.Ss

Multi-component Fermi gases with tunable interactions are exceptionally well suited to the study of few- and many-body quantum physics. Ultracold twostate Fermi gases near a Feshbach resonance have been used to characterize the crossover from Bardeen-CooperSchreiffer (BCS) superfluidity to Bose-Einstein condensation (BEC) of diatomic molecules [1]. Further, a normal to superfluid transition and phase separation have been observed in imbalanced two-state spin mixtures [2]. The stability of the two-state mixtures against two- and three-body loss processes was critically important to the success of these experiments.

Recently, there has been considerable interest in the study of three-state Fermi gases with tunable interactions [3, 4, [5, 6, 6, 7] $]$. Whereas three-body interactions in ultracold two-state Fermi gases are suppressed by the exclusion principle, the addition of a third spin component allows for the study of three-body phenomena such as the Efimov effect (7). Further, with this additional spin state there may be competition between multiple pairing states and trion formation [3, 4, 흐. For example, if pairwise interactions are all attractive and of equal magnitude, the system is expected to exhibit a novel superfluid phase analogous to color superconductivity in quantum chromodynamics (QCD) [5]. A quantum phase transition to a Fermi liquid of trimers (analogous to baryons) may be observed if the ratio of the interaction energy to the kinetic energy is increased (e.g. by an optical lattice) [5].

Future studies of the above phenomena critically depend on the magnitude of two- and three-body loss rates, particularly when two or more scattering lengths are resonantly enhanced. Two- and three-body loss and heating processes can impose stringent limits on the maximum achievable phase space density. Further, the unambiguous observation of three-body resonances requires negligible two-body loss 9 ].

Most investigations of Fermi gases with tunable interactions have focussed on two-state mixtures. A small admixture of a third spin component has been used for thermometry 10] and recently the rapid decay of a three-state Fermi gas near a Feshbach resonance was noted during an investigation of Cooper pair size by radio-frequency (RF) spectroscopy in a two-state Fermi gas 11]. Very recently our group reported measurements of three-body loss in thermal and degenerate three-state Fermi gases of ${ }^{6} \mathrm{Li}$ for magnetic fields between 400 and $960 \mathrm{G}$ which included three Feshbach resonances [12]. Independently, Ottenstein et al. observed loss in a threestate ${ }^{6} \mathrm{Li}$ gas for field values between 0 - $750 \mathrm{G}$ (including a single Feshbach resonance) and reported three-body loss rate coefficients for field values up to $600 \mathrm{G}$ (excluding any pairwise scattering resonances) [13].

In this Letter, we report the first measurements of three-body loss coefficients for a three-state Fermi gas over a range of magnetic fields where pairwise interactions are resonantly enhanced. This enhancement is due to three overlapping Feshbach resonances and a zeroenergy resonance in the ${ }^{6} \mathrm{Li}$ triplet molecular potential. Further, we demonstrate that all two-state mixtures of the three lowest energy hyperfine states of ${ }^{6} \mathrm{Li}$ are stable against two-body loss processes for almost the entire range of field values between 15 and $953 \mathrm{G}$, making this three-state mixture well suited to studies of three-body physics. We observe a narrow loss feature at $127 \mathrm{G}[14$ which may be due to a three-body resonance and an additional state-dependent loss feature at $504 \mathrm{G}$. Finally, we measure the three-body loss rate coefficient at high fields where all three scattering lengths are asymptoting to the triplet scattering length $a_{t}=-2140 a_{0}$. This measurement and an observed trend toward lower loss at higher fields has important implications for realizing cold atom analogs of color superfluid and baryon phases in QCD.

We study a ${ }^{6} \mathrm{Li}$ Fermi gas with equal populations in the three lowest energy hyperfine states. At zero field, the three states correspond to $|1\rangle=\left|\frac{1}{2},+\frac{1}{2}\right\rangle,|2\rangle=\left|\frac{1}{2},-\frac{1}{2}\right\rangle$ and $|3\rangle=\left|\frac{3}{2},-\frac{3}{2}\right\rangle$ in the $\left|f, m_{f}\right\rangle$ basis. For fields above $\simeq 200 \mathrm{G}$, these states become increasingly electron-spin polarized and primarily differ by their nuclear spin pro- 


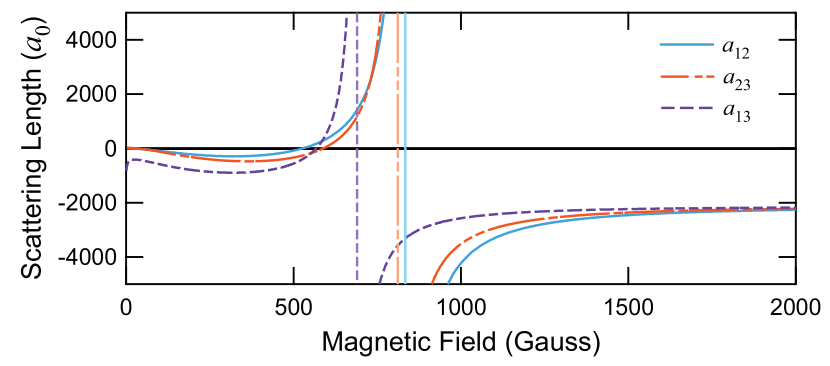

FIG. 1: (color online) The $s$-wave scattering lengths as a function of magnetic field for interactions between the three lowest energy hyperfine ground states of ${ }^{6} \mathrm{Li}(|1\rangle,|2\rangle$ and $|3\rangle)$ [15]. Feshbach resonances occur at 690, 811, and $834 \mathrm{G}$.

jection. The pairwise $s$-wave scattering lengths between these states $\left(a_{12}, a_{23}\right.$, and $\left.a_{13}\right)$ exhibit three broad overlapping Feshbach resonances as shown in Fig. 11 15]. For very large magnetic fields, each of the pairwise scattering lengths asymptote to the triplet scattering length for ${ }^{6} \mathrm{Li}$, $a_{t}=-2140 a_{0}$. Therefore, in the limit of large magnetic fields, the interactions are $\mathrm{SU}(3)$ symmetric [5].

Two-body spin-flip processes are expected to be especially small for this ultracold ${ }^{6} \mathrm{Li}$ mixture. Spin-exchange collisions are energetically forbidden in a magnetic field. Dipolar relaxation by electron spin-flip is suppressed at high field as the gas becomes increasingly electron-spin polarized in the lowest energy electron spin state.

We can produce a degenerate Fermi gas (DFG) with an equal mixture of atoms in states $|1\rangle$ and $|2\rangle$ once every 5 seconds by evaporatively cooling this mixture in a red-detuned optical dipole trap [16]. During the first second, $\sim 10^{8}{ }^{6} \mathrm{Li}$ atoms from a Zeeman-slowed atomic beam are collected in a magneto-optical trap (MOT). A crossed optical dipole trap which overlaps the MOT is then turned on and optimally loaded by tuning the MOT laser beams $\simeq 6 \mathrm{MHz}$ below resonance and reducing their intensity for $7 \mathrm{~ms}$. The atoms are then optically pumped into states $|1\rangle$ and $|2\rangle$ prior to extinguishing the MOT laser beams and field gradient. The two beams which form the optical trap derive from a linearly polarized multi-longitudinal-mode $110 \mathrm{~W}$ fiber laser operating at $1064 \mathrm{~nm}$. The beams nearly co-propagate in the vertical direction $(\hat{y})$, have orthogonal polarizations and cross at an angle $\simeq 11^{\circ}$. The beams are elliptical with calculated $e^{-2}$ waist radii $\sim 30 \mu \mathrm{m}$ and $\sim 100 \mu \mathrm{m}$ at the point of intersection. The maximum trap depth per beam is $\simeq 1 \mathrm{mK}$ allowing $\sim 5 \times 10^{6}$ atoms to be initially loaded. We apply a bias field and a noisy RF pulse to create a 50-50 mixture of atoms in states $|1\rangle$ and $|2\rangle$ [16].

Forced evaporation of the atoms occurs at a field of $330 \mathrm{G}$ where the two-state scattering length $a_{12} \simeq$ $-280 a_{0}$. We lower the depth of the optical trap $U_{0}$ by a factor of 107 over 3.6 seconds to obtain our final temperature. For the work presented here we study a gas at a temperature $T \gtrsim 0.5 T_{F}$ ( $T_{F}$ is the Fermi temperature) so that it is appropriate to treat the cloud as a thermal

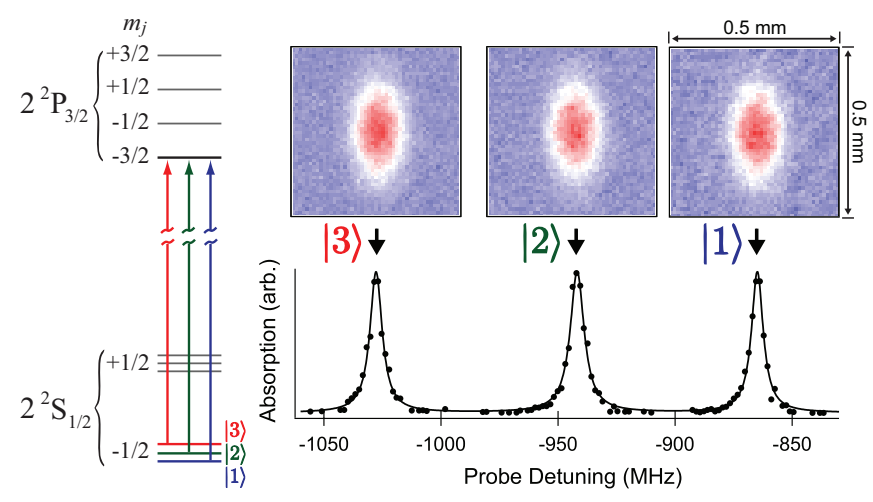

FIG. 2: (color online) The population in states $|1\rangle,|2\rangle$ and $|3\rangle$ measured by absorption imaging in the Paschen-Back regime at a bias field of $568 \mathrm{G}$ [18]. A $\sigma^{-}$polarized probe beam drives $m_{j}=-\frac{1}{2} \rightarrow m_{j^{\prime}}=-\frac{3}{2}$ transitions which, due to the hyperfine interaction in the ground state, are spectroscopically resolvable. (Top right) The on-resonance absorption images show the density profile for the three states following a $900 \mu \mathrm{s}$ time-of-flight expansion. (Bottom right) The total absorption vs. probe detuning (relative to the $\left|f=\frac{1}{2}, m_{f}=-\frac{1}{2}\right\rangle \rightarrow$ $\left|f^{\prime}=\frac{3}{2}, m_{f^{\prime}}=-\frac{3}{2}\right\rangle$ transition frequency in zero field).

gas in the analysis. To suppress further loss by evaporation for the remainder of the experiment, $U_{0}$ is increased by a factor of 4 increasing the ratio of $U_{0} / k_{B} T$ by a factor $\simeq 2$. The final oscillation frequencies of the trap are $\nu_{x}=3.84 \mathrm{kHz}, \nu_{y}=106 \mathrm{~Hz}$ and $\nu_{z}=965 \mathrm{~Hz}$ [17] with a final trap depth per beam $\simeq 40 \mu \mathrm{K}$. The total number of atoms $\simeq 3.6 \times 10^{5}$ in a balanced two-state mixture at $T \simeq 1.9 \mu \mathrm{K}$ for which $T_{F} \simeq 3.7 \mu \mathrm{K}$. The background limited $1 / e$-lifetime $\simeq 30 \mathrm{~s}$.

To create an incoherent three-state mixture we first increase the strength of the magnetic field in $10 \mathrm{~ms}$ to $568 \mathrm{G}$ where the mixture is stable. We then apply a noisy RF magnetic field with two frequencies centered on the $|1\rangle-|2\rangle$ and $|2\rangle-|3\rangle$ transitions (each with a power corresponding to an on-resonance Rabi frequency $\Omega \sim$ $2 \pi \times 18 \mathrm{kHz}$ and both broadened to a width of $1 \mathrm{MHz}$ ). A magnetic field gradient of $\simeq 1 \mathrm{G} / \mathrm{cm}$ in the direction of the bias field $(\hat{z})$ is simultaneously applied to destroy any internal state coherence in the sample. The noisy RF field remains on for $50 \mathrm{~ms}$ to create an equal mixture of $N \simeq$ $1.2 \times 10^{5}$ atoms in each state. At this point, $T=1.9 \mu \mathrm{K}$ with $T_{F}=3.2 \mu \mathrm{K}$ and the peak density in a single spin state is $5.5 \times 10^{12}$ atoms $/ \mathrm{cm}^{3}$. We measure the population and temperature of atoms in each state separately by spectroscopically-resolved absorption imaging (Fig. 2).

To investigate the magnetic field dependence of decay in this three state mixture, we compared the number of atoms remaining in each spin state after spending two different times, $1 \mathrm{~ms}$ and $201 \mathrm{~ms}$, at a particular field of interest $B$ (see Fig. $3(\mathrm{a})$ ). For each experimental cycle the magnetic field was ramped in $10 \mathrm{~ms}$ from $568 \mathrm{G}$ (where the mixture was first created) to the field of interest where it was held constant for either $1 \mathrm{~ms}$ or $201 \mathrm{~ms}$. 


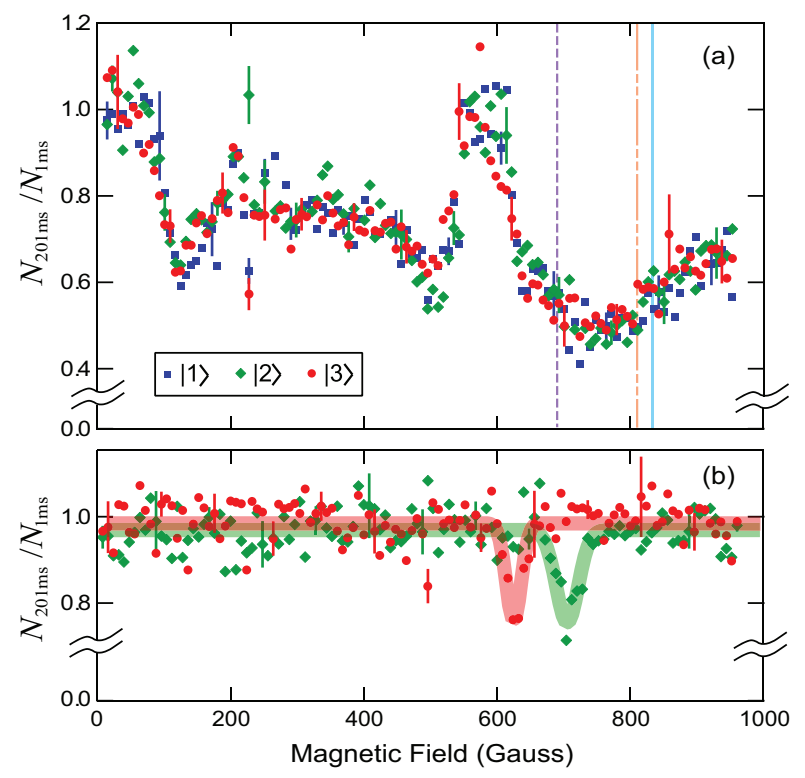

FIG. 3: (a) Number remaining in each of the three spin states after spending $201 \mathrm{~ms}$ at the field of interest $B$ (normalized to the number remaining after spending $1 \mathrm{~ms}$ at $B$ ). (b) Magnetic field dependence of decay in individual two-state mixtures. $(\bullet)$ indicates the normalized number remaining in state $|2\rangle(|3\rangle)$ of a $|2\rangle-|3\rangle(|1\rangle-|3\rangle)$ mixture after $201 \mathrm{~ms}$. The thick lines guide the eye. The representative error bars indicate the standard deviation in the mean. Absorption images are taken at $953 \mathrm{G}$ to ensure that any weakly-bound molecules which remain trapped are dissociated and measured.

The field was then ramped in $10 \mathrm{~ms}$ to $953 \mathrm{G}$ and held for $20 \mathrm{~ms}$ before absorption imaging at $953 \mathrm{G}$ to measure the atom number $\left(N_{1 \mathrm{~ms}}\right.$ or $\left.N_{201 \mathrm{~ms}}\right)$. The $10 \mathrm{~ms}$ field ramp to $953 \mathrm{G}$ (where $a_{12}, a_{23}, a_{13}<0$ ) ensured that any atoms which had formed weakly bound molecules in the vibrational state associated with the Feshbach resonances (but remained trapped) would be dissociated and measured. For each field value, $N_{201 \mathrm{~ms}}$ is normalized by $N_{1 \mathrm{~ms}}$ to correct for the fraction of atoms lost during the field sweeps, both to and from the field of interest $B$, which exhibited a slowly varying dependence on $B$. See Ref. 21] for the data before normalization.

To confirm that two-body loss does not contribute significantly to the measurements described above, we measured the ratio of atoms remaining after evolution times of $201 \mathrm{~ms}$ and $1 \mathrm{~ms}$ at fixed magnetic field values for each of the three possible two-state mixtures. Figure 3(b) shows the ratio $N_{201 \mathrm{~ms}} / N_{1 \mathrm{~ms}}$ for atoms in state $|3\rangle$ (state $|2\rangle)$ of a $|1\rangle-|3\rangle(|2\rangle-|3\rangle)$ mixture. Data for the $|1\rangle-|2\rangle$ mixture (not shown) is very similar to that of the $|2\rangle-|3\rangle$ mixture (e.g. Ref. [19]). With the exception of loss features between 600 and $750 \mathrm{G}$, each two-state mixture was stable. The loss features are due to three-body recombination to bound molecular states associated with the Feshbach resonance. Since the two-state mixtures are stable for fields $\leq 600 \mathrm{G}$ and $\geq 750 \mathrm{G}$, loss features observed in three-state mixtures at these fields are due to three-body events involving one atom from each spin state.

In Fig. 3(a), the broad dominant loss feature centered at $720 \mathrm{G}$ occurs in the vicinity of three overlapping interspecies Feshbach resonances. Significant loss due to three-body recombination is expected near these resonances since recombination events involving one atom in each spin state are not suppressed by the exclusion principle and one expects a significant increase in the event rate when two or more scattering lengths are resonantly enhanced [20]. Similarly, high stability at zero field and near the zero crossings of the Feshbach resonances is not surprising since at least two of the scattering lengths are small at these fields. At high field, the stability increases relative to that at $720 \mathrm{G}$.

Unexpected resonant loss features are observed at 127 and $504 \mathrm{G}$, where the two-body scattering lengths are not predicted to exhibit any resonances. We have observed these features at higher temperatures and in a different trap configuration [21]. A possible explanation for the feature at $127 \mathrm{G}$ is that the binding energy of a three-body bound state crosses through zero near this field value. At $504 \mathrm{G}$, we observe differing degrees of enhanced loss for the three states leading to population imbalance. This resonance was not observed in Ref. [13].

The feature at $228 \mathrm{G}$ in Fig. 3 is consistent with our estimated location of a $|1\rangle-|3\rangle$-wave Feshbach resonance which would yield the observed preferential loss of atoms from states $|1\rangle$ and $|3\rangle$ [22]. We also observed a very narrow but inconsistent loss feature at $259 \mathrm{G}$ (not shown). The modest increase in stability observed near $200 \mathrm{G}$ did not appear in our other experimental data [21].

A second set of experiments measured three-body loss coefficients at fixed magnetic fields where the decaying populations remained balanced. In these experiments, the magnetic field is swept to the field of interest $B$ in $10 \mathrm{~ms}$ after creation of the three-state mixture and the number $N(t)$ and temperature $T(t)$ of atoms in state $|3\rangle$ are then measured at the field $B$ for various delay times $t$. To extract the three-body loss coefficients, we fit this data assuming one- and three-body loss and heating due to three-body recombination.

When three-body recombination involves one atom from each spin state, the number of trapped atoms in each of the equally populated spin states $N(t)$ evolves according to $\dot{N}=-L_{1} N-L_{3}\left\langle n^{2}\right\rangle N$. Here, $\left\langle n^{2}\right\rangle$ is the average value of the squared density per spin state and $L_{3}\left(L_{1}\right)$ is the three-body (one-body) atom-loss rate coefficient. The density distribution in the trap is well described by a thermal distribution so that

$$
\frac{d N}{d t}=-L_{1} N-\gamma \frac{N^{3}}{T^{3}}
$$

where $\gamma=L_{3}\left(m \bar{\omega}^{2} / 2 \pi k_{B}\right)^{3} / \sqrt{27}$ and $\bar{\omega}=\left(\omega_{x} \omega_{y} \omega_{z}\right)^{1 / 3}$. Following Ref. [23], we model the temperature increase in the gas as arising from "anti-evaporation" and recom- 


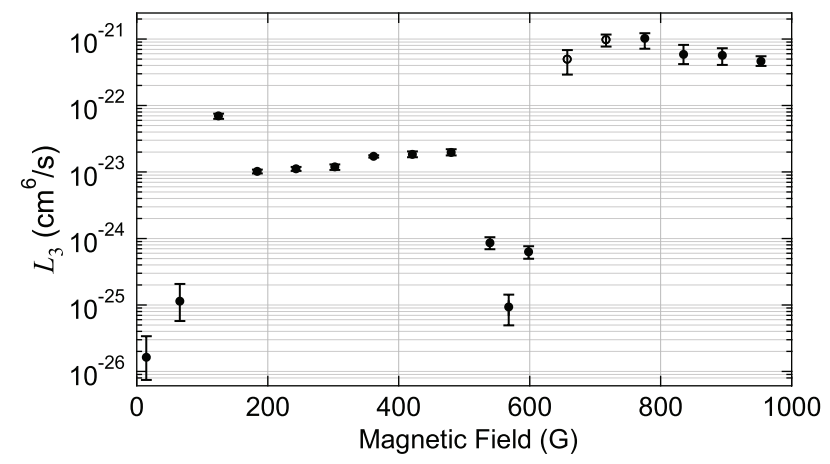

FIG. 4: Three-body loss rate coefficient, $L_{3}$, at various fields.

bination heating. The temperature evolves as

$$
\frac{d T}{d t}=\gamma \frac{N^{2}}{T^{3}} \frac{\left(T+T_{h}\right)}{3},
$$

where $k_{B} T_{h}$ is the average energy deposited in the gas per recombination event.

For a given parameter set $\left(N(0), T(0), L_{1}, L_{3}\right.$ and $T_{h}$ ), Eqns. 10 and 2 can be numerically integrated to give $N(t)$ and $T(t)$. The best-fit parameters for a data set at a given field are determined by minimizing $\chi^{2}$. The bestfit $L_{3}$ values at various fields are displayed in Fig. 4 For these fits, $T_{h}$ varied from $1.5-4 \mu \mathrm{K}$ and $L_{1}$ varied from $0.1-0.33 \mathrm{~s}^{-1}$. The error bars in Fig. 4 indicate the $68 \%$ confidence interval due to statistical uncertainty in all fit parameters. The error bars do not include a systematic uncertainty of $\pm 70 \%$ arising from our uncertainty in atom number $( \pm 30 \%)$ and trap frequencies $( \pm 5 \%)$.

As shown in Fig. 4. $L_{3}$ varies by over four orders of magnitude. In the range $600 \mathrm{G}<B<750 \mathrm{G}$, the $L_{3}$ values (o) may be overestimated since the measured decay includes loss events we observe in two-state mixtures at these field values. Near 0 and $568 \mathrm{G}$, three-body recombination rates are relatively small and stable three-state Fermi gases can be created. For $L_{3}=10^{-25} \mathrm{~cm}^{6} / \mathrm{s}$, a gas with a density $n=10^{12} \mathrm{~cm}^{-3}$ per spin state has a $1 / e$-lifetime $\sim 10$ seconds. Consistent with the data shown in Fig. [3(a), a resonant peak in $L_{3}$ is observed at $B \simeq 127 \mathrm{G}$. We do not report a value for $L_{3}$ at the location of the $504 \mathrm{G}$ resonant feature (Fig. 3(a)) since a population imbalance develops there. The highest $L_{3}$ value we report $\left(10^{-21} \mathrm{~cm}^{6} / \mathrm{s}\right)$ occurs at $775 \mathrm{G}$ and is likely unitarity limited (for fields $>600 \mathrm{G}, T \simeq 6 \mu \mathrm{K}$ due to heating). We observe that $L_{3}$ decreases by a factor of $\simeq 2.5$ as the field is increased from 775 to $953 \mathrm{G}$, the highest field we currently access. This trend suggests that $L_{3}$ may decrease further in the high field limit where $a_{12}, a_{23}, a_{13} \rightarrow-2140 a_{0}$.

In conclusion, we can create long-lived three-state DFGs of ${ }^{6} \mathrm{Li}$ atoms for fields near $568 \mathrm{G}$. The minimal two-body loss we observe makes this system promising for future studies of Efimov trimers. Indeed, the loss resonances at 127 and $504 \mathrm{G}$ may be occurring at field values where trimer states cross the dissociation threshold. The recombination rates at high fields need not preclude experiments designed to study color superfluidity and trion formation 5]. For instance, using low density gases $\left(n \sim 5 \times 10^{10} \mathrm{~cm}^{-3}\right)$ and large period $(d \simeq 2 \mu \mathrm{m})$ optical lattices, long lifetimes ( $\gtrsim 0.1 \mathrm{~s})$ and strong interactions can simultaneously be achieved.

This material is based upon work supported by the AFOSR (Award FA9550-08-1-0069), the ARO (Award W911NF-06-1-0398) and the NSF (PHY 07-01443).

[1] C. A. Regal et al., Phys. Rev. Lett. 92, 040403 (2004); M. W. Zwierlein et al., Phys. Rev. Lett. 92, 120403 (2004); J. Kinast et al., Phys. Rev. Lett. 92, 150402 (2004); C. Chin et al., Science 305, 1128 (2004); T. Bourdel et al., Phys. Rev. Lett. 93, 050401 (2004); M. W. Zwierlein et al., Nature 435, 1047 (2005); G. B. Partridge et al., Phys. Rev. Lett. 95, 020404 (2005).

[2] G. B. Partridge et al., Science 311, 503 (2006); G. B. Partridge et al., Phys. Rev. Lett. 97, 190407 (2006); M. W. Zwierlein et al., Science 311, 492 (2006); Y. Shin et al., Phys. Rev. Lett. 97, 030401 (2006); M. W. Zwierlein et al., Nature 442, 54 (2006).

[3] A. G. K. Modawi and A. J. Leggett, Jour. Low. Temp. Phys. 109, 625 (1997); C. Honerkamp and W. Hofstetter, Phys. Rev. B 70, 094521 (2004); T. Paananen et al., Phys. Rev. A 73, 053606 (2006); P. F. Bedaque et al., arXiv:cond-mat/0602525 (2006).

[4] L. He et al., Phys. Rev. A 74, 033604 (2006).

[5] A. Rapp et al., Phys. Rev. Lett. 98, 160405 (2007).

[6] H. Zhai, Phys. Rev. A 75, 031603(R) (2007); R. W. Cherng et al., Phys. Rev. Lett. 99, 130406 (2007); X. W. Guan et al., Phys. Rev. Lett. 100, 200401 (2008); X.-J. Liu et al., Phys. Rev. A 77, 013622 (2008).

[7] E. Braaten and H.-W. Hammer, Ann. Phys. 322, 120 (2007); T. Luu and A. Schwenk, Phys. Rev. Lett. 98, 103202 (2007).

[8] S. Capponi et al., Phys. Rev. A 77, 013624 (2008).

[9] T. Kraemer et al., Nature 440, 315 (2006).

[10] C. A. Regal, Ph.D. thesis, CU-Boulder (2005).

[11] C. H. Schunck et al., Nature 454, 739 (2008).

[12] J. Williams et al., Bull. Am. Phys. Soc. 53, 80 (2008).

[13] T. B. Ottenstein et al., Phys. Rev. Lett. 101, 203202 (2008).

[14] This resonance was first reported in Ref.[13].

[15] M. Bartenstein et al., Phys. Rev. Lett. 94, 103201 (2005).

[16] K. M. O'Hara et al., Science 298, 2179 (2002).

[17] We determine trap frequencies by observing dipole oscillations $\left(\omega_{y}\right)$ and parametric excitation $\left(\omega_{x}, \omega_{z}\right)$.

[18] Fields are calibrated to $\pm 1 \mathrm{G}$ by $\mathrm{RF}$ /optical spectroscopy.

[19] K. Dieckmann et al., Phys. Rev. Lett. 89, 203201 (2002).

[20] Three particles strongly interact even if one of the pairwise scattering lengths is small since the third particle acts as a mediator for the weakly interacting pair.

[21] See EPAPS Document No. E-PRLTAO-102-027919 for additional data. For more information on EPAPS, see http://www.aip.org/pubservs/epaps.html

[22] Estimate based on $p$-wave resonances in C. H. Schunck et al., Phys. Rev. A 71, 045601 (2005).

[23] T. Weber et al., Phys. Rev. Lett. 91, 123201 (2003). 